Published in final edited form as:

Nat Rev Neurol. 2014 May ; 10(5): 271-282. doi:10.1038/nrneurol.2014.64.

\title{
SUDEP Mechanisms: The pathway to prevention
}

\author{
Cory A. Massey ${ }^{\star}{ }^{1,2}$, Levi P. Sowers ${ }^{\star}, 2$, Brian J. Dlouhy ${ }^{3}$, and George B. Richerson ${ }^{1,2,4,5}$ \\ ${ }^{1}$ Interdisciplinary Graduate Program in Neuroscience, 200 Hawkins Drive, 2 RCP, University of \\ lowa, lowa City, IA 52242 \\ ${ }^{2}$ Department of Neurology, 200 Hawkins Drive, 2 RCP, University of lowa, lowa City, IA 52242 \\ ${ }^{3}$ Department of Neurosurgery, 200 Hawkins Drive, 2 RCP, University of lowa, lowa City, IA 52242 \\ ${ }^{4}$ Department of Molecular Physiology \& Biophysics, 200 Hawkins Drive, 2 RCP, University of \\ lowa, lowa City, IA 52242
}

${ }^{5}$ VA Medical Center, lowa City, IA 52242

\begin{abstract}
Sudden unexpected death in epilepsy (SUDEP) is the leading cause of death in patients with refractory epilepsy, and yet many physicians do not know about this high risk of sudden death. It has been widely believed that SUDEP is due to cardiac abnormalities during the post-ictal period. However, recent studies have demonstrated that respiratory depression is common following a seizure, and can be severe enough to cause a substantial decrease in oxygen saturation. In this review we summarize evidence for cardiac, respiratory, and arousal abnormalities during the ictal and post-ictal period and potential mechanisms for these abnormalities. We discuss mouse models of seizure-induced death and how these models are useful for understanding the mechanisms that underlie SUDEP. Some of these are due to genetic mutations that have counterparts in human syndromes. Controversy remains regarding the relative importance of cardiac failure versus respiratory arrest as the primary cause of death. Resolving this controversy will require simultaneous monitoring of cardiac and respiratory parameters during cases of near SUDEP in humans and detailed pathophysiological data from animal models during seizure-induced death. Effective preventive strategies in high-risk patients will rely on defining the mechanisms that initiate the sequence of events that lead from seizures to death.
\end{abstract}

\section{Introduction}

Most patients with epilepsy lead a normal life. As long as they take their medication, a majority can remain seizure free, work, and be active. If they do have a seizure, the risk of injury is usually minimal as long as they are not driving or in another dangerous situation. However, in recent years it has become increasingly appreciated that patients with epilepsy have a risk of sudden death that is more than 20 times greater than the general population1. This SUDEP is estimated to cause 2000-3000 deaths per year in the U.S. alone ${ }^{2}$, which is probably an underestimate for reasons described below, and accounts for as many as $15 \%$ of

\footnotetext{
*These authors made equal contributions
} 
all epilepsy related deaths ${ }^{3}$. The single risk factor identified most consistently across multiple studies is the frequency of generalized tonicclonic seizures (GTCS) ${ }^{4}$.

Despite such a high incidence, in Canadian pediatricians who treat children with epilepsy, only $56 \%$ reported that they knew their patients were at higher risk of sudden death. Furthermore, only $33 \%$ of the surveyed pediatricians had heard of the term SUDEP ${ }^{5}$. These numbers are likely similar in the US, and are probably higher among non-neurologists. However, these numbers will likely change rapidly, because SUDEP is becoming more salient among patients and their families, and as they educate themselves doctors will become more aware. The pace of publications on SUDEP is rapidly increasing ${ }^{6}$, yet there remains a gap in our knowledge of the mechanisms involved, in part because the unpredictable occurrence of SUDEP has hindered the gathering of high quality data at the time of death. In particular, there have been human SUDEP cases in which the EKG was recorded and respiratory rate was estimated by visual observation, but no blood pressure or tidal volume data were obtained. Here we discuss data from SUDEP cases, experiments monitoring human peri-ictal physiology and recordings during seizure-induced death in animal models. We then interpret these data and their relevance to the mechanisms of SUDEP.

SUDEP is "the sudden, unexpected, witnessed or unwitnessed, non-traumatic, and nondrowning death of patients with epilepsy with or without evidence of a seizure, excluding documented status epilepticus, and in which postmortem examination does not reveal a structural or toxicological cause of death"7. There is no diagnostic test for SUDEP, and no finding on autopsy that is pathognomonic. Therefore, ascertainment of cases is difficult, because the term SUDEP is not always used by coroners or medical examiners. There is also significant variability in the patient populations included in epidemiological studies. These factors contribute to the wide variation in estimates of the incidence of SUDEP, ranging from as little as 0.09 per 1000 persons per year among unselected incident cases, all the way to 9.3 per 1000 persons per year in surgical resection candidates 3. Misclassification may also contribute to the wide range of published estimates for SUDEP incidence. For example, among refractory epilepsy patients SUDEP has been determined to account for as little as $10 \%$ to as much as $50 \%$ of all deaths ${ }^{3}$. A recent meta-analysis from the CDC of refractory patients has placed their lifetime risk for SUDEP at $37 \%^{2}$. Although the total number of SUDEP deaths is not as large as that of many other neurological disorders, such as Parkinson's Disease, Alzheimer's Disease and motor neuron disease, the peak incidence of SUDEP occurs at the age of only 30 (Fig. 1), so the "years of potential life lost" is second only to stroke among all neurological diseases ${ }^{2}$.

\section{Pathophysiology}

Determining the pathophysiological mechanisms underlying SUDEP is critical in order to develop effective preventive treatments. It has been well known for many years that seizures can induce cardiac arrhythmias (Fig. 2a), changes in blood pressure, and asystole, and this led to the assumption that SUDEP was due to a cardiac mechanism ${ }^{8}$. However, respiratory depression and apnea occuring during and after a seizure ${ }^{9-11}$ have been well documented, and these abnormalities can be severe enough to cause oxygen desaturation (Fig. 2b) ${ }^{12,13}$. 
These observations led to a debate about whether the primary, or initiating, event in SUDEP is more frequently an abnormality of breathing, cardiovascular function, or both. Some reports have hypothesized that pulmonary edema is a possible cause of death, as mild pulmonary edema is regularly found in SUDEP cases upon autopsy ${ }^{14-16}$. However, this mild edema is not considered severe enough to be the primary cause of death ${ }^{3}$. Here we will focus the effect of seizures on breathing and cardiac function. We will then describe how the loss of arousal mechanisms impairs the protective reflexes that can be essential for survival during recovery from a seizure. Finally, we will discuss insights obtained about mechanisms from SUDEP cases that were monitored at the time of death.

\section{Seizures and respiratory dysfunction}

Lack of oxygenation during seizures was first reported in 1899 when Hughlings Jackson noted his human patients and monkeys "turning blue" during seizures ${ }^{9}$. Since then, others have noted similar findings, and have documented apnea ${ }^{10,17}$ and oxygen desaturation ${ }^{11-13}$ during and after seizures. Using long-term video-EEG monitoring and respiratory recordings, Nashef and colleagues ${ }^{12}$ found that 10 of 17 patients (59\%) developed apnea and 6 patients experienced oxygen desaturation $\left(\mathrm{SaO}_{2}\right)$ ranging from 55-83\%, both concurrent with seizures ${ }^{12}$. Investigating 304 seizures in 56 patients with intractable localization-related epilepsy, Bateman and colleagues ${ }^{13}$ reported $\mathrm{SaO}_{2}$ values decreasing below $90 \%$ in $33.2 \%$ of seizures, below $80 \%$ in $10.2 \%$ of seizures, and below $70 \%$ in $3.6 \%$ of seizures.

Desaturation was common and severe even with seizures that did not generalize. In 100 seizures for which data were available including airflow measurements, central apnea or hypopnea occurred in 50\%, whereas mixed or obstructive apneas occurred in $9 \%$ of seizures ${ }^{13}$. In seven patients with 19 seizures for which end-tidal carbon dioxide $\left(\mathrm{ETCO}_{2}\right)$ data were available, $\mathrm{SaO}_{2}$ below $85 \%$ was accompanied by an increase in $\mathrm{ETCO}_{2}$ of $18.6 \pm$ $17.7 \mathrm{mmHg}$, indicating hypoventilation ${ }^{13}$. Oxygen desaturation was not unique to GTCS, as $\mathrm{SaO}_{2}$ decreased below $90 \%$ in $34 \%$ of partial seizures ${ }^{13}$. These data provide important evidence that respiratory depression could be severe enough to cause SUDEP, but they raise the question of why death is not even more common given how often seizures induce hypoventilation.

Peri-ictal hypoventilation can lead to secondary cardiac failure. When $\mathrm{SaO}_{2}$ drops below $90 \%$, it has been shown that there is a greater than four-fold likelihood of QT prolongation as compared to seizures without decreased $\mathrm{SaO}_{2}{ }^{18}$. Moreover, in seizures with oxygen desaturatinon there is a greater than two fold increase in likelihood that the QT interval is shortened. These findings raise a cautionary note, because cardiac changes observed as terminal events in SUDEP cases may be due to hypoventilation that would go unnoticed without proper monitoring.

Animal models are a valuable way to determine underlying mechanisms of seizure-induced death. DBA/2 mice are a well-established mouse model of audiogenic seizures, which lead to respiratory arrest and death, but the mice can be resuscitated using a ventilator ${ }^{19}$. A caveat is that these mice do not have spontaneous seizures so they would not meet the definition of SUDEP, which requires that the subject has epilepsy. However, there are no data to show that epilepsy is required for a seizure to be fatal in humans, rather than death 
being due to the seizure itself. Data from DBA/2 mice indicate that seizures inhibit the ponto-medullary respiratory control network responsible for control of breathing ${ }^{20,21}$, which leads to apnea followed by asystole and death. Seizure-induced respiratory arrest and death also occurs in DBA/1 mice, HTR2C knockout mice, and others ${ }^{22,} 23$. Seizures can produce a strong inhibitory effect on breathing, and in mouse models this can be fatal, but it remains unclear whether this can occur in humans.

\section{Seizures and cardiac dysfunction}

In 1906 cardiac asystole during a seizure was reported by Russell ${ }^{24}$. Since then many ictal cardiac abnormalities have been reported such as a prolonged QT interval, tachycardia, bradycardia, and Torsades de Pointes ${ }^{8,25}$. Seizures often induce tachyarrhythmias, with $76 \%$ of epilepsy patients experiencing tachycardia during at least one seizure, and $57 \%$ of seizures are accompanied by tachycardia. This is closely correlated with seizure generalization ${ }^{26,27}$, and may be due to seizure spread to the hypothalamus with activation of first order sympathetic neurons. The same studies found that only $2 \%$ of seizures result in bradycardia, while only $0.5 \%$ resulted in asystole. Moreover, in a study with over 250 seizures in 56 patients, one case of peri-ictal bradycardia was reportedly followed by asystole ${ }^{13}$. This case had concurrent $\mathrm{O}_{2}$ saturation that fell below $50 \%$ suggesting that bradycardia and asystole may have been secondary to hypoventilation. Two additional reports demonstrate that hypercapnia and hypoxia can both lead to tachycardia in subjects with no cardiac abnormalities ${ }^{28,29}$, consistent with known effects of the respiratory system on autonomic output. These can be due to changes in blood gases or due to interactions between neurons with the brainstem.

Repolarization abnormalities such as QT prolongation are common during seizures and it is proposed that these could contribute to SUDEP pathophysiology. Recent studies have found that there is prolongation of the QT interval during seizures. In a study of 76 patients, significant ictal prolongation of the QT interval was found in $4.8 \%$ of seizures whereas short QT intervals were observed in $3.8 \%$ of seizures ${ }^{30}$. However, no SUDEP risk factors were associated with altered QT intervals.

Heart rate variability (HRV) is due to modulation of the sinoatrial node by the autonomic nervous system ${ }^{31}$. Patients with temporal lobe epilepsy (TLE) have reduced HRV when compared to the general population, and this reduction is more pronounced during the night ${ }^{32,33}$. Reports show abnormalities in HRV and cerebrovascular autoregulation could be due to seizure activity, since they improve after epilepsy surgery 34,35 . HRV defects are correlated with surgical outcome - patients with the poorest outcome have more significant HRV impairment ${ }^{36}$. Reduced HRV has been associated with increased cardiac mortality and sudden cardiac death ${ }^{31,37}$. Therefore, it would be expected that HRV is involved in SUDEP mechanisms. Others have reported an inverse correlation between high frequency HRV and risk of SUDEP ${ }^{38}$. However, a study comparing 7 SUDEP cases and 7 age-matched controls showed no difference in $\mathrm{HRV}^{39}$. Therefore, the role HRV plays in SUDEP pathophysiology remains unclear. 


\section{Post-ictal arousal deficits}

GTCS $^{40,41}$ and complex partial seizures (CPS $)^{42,43}$ are both associated with behavioral arrest and impaired consciousness. Patients with these seizures are unresponsive to commands and questions during the post-ictal state, however patients with CPS are able to grasp a ball and visually track ${ }^{44}$. These data suggest that although consciousness is reduced in CPS, they are closer to a minimally conscious state than a post-ictal coma as occurs following GTCS, which could increase risk of SUDEP.

Work in the 1930s and 1950s by Wilder Penfield and Herbert Jasper led to the theory that control of consciousness involved subcortical structures based in the diencephalon and upper brainstem ${ }^{45,46}$. These subcortical structures responsible for arousal and consciousness are collectively known as the ascending arousal system (AAS) $)^{47,48}$. This system is composed of discrete neurotransmitter-specific nuclei, including the raphe nuclei ${ }^{49}$, the locus coeruleus ${ }^{50}$, the ventral tegmental area $^{51}$, the pedunculopontine and laterodorsal tegmental nuclei ${ }^{52}$, and the parabrachial complex ${ }^{53}$. If a seizure spreads into the midbrain and medulla and affects these nuclei it could alter a patient's level of consciousness. Blumenfeld ${ }^{54}$ proposed the network inhibition hypothesis, which posits that consciousness is maintained through interactions between the cortex and subcortical structures. Once aberrant discharges start in the cortex, they travel along known anatomical connections to subcortical structures and activate GABAergic interneurons that inhibit the AAS. With the AAS inhibited, cortical activity is suppressed resulting in loss of consciousness. Data supports this theory, as SPECT imaging has shown increased blood flow starting in temporal lobe structures and descending into the midbrain and brainstem in patients with temporal-lobe CPS ${ }^{55}$. Furthermore, recordings from cholinergic neurons in the pedunculopontine tegmental nucleus demonstrate that acetylcholine neurons in the AAS are inhibited during limbic seizures ${ }^{56}$. Loss of consciousness due to AAS inhibition leaves a patient with epilepsy at greater risk for SUDEP, as protective reflexes are suppressed during the post-ictal coma.

\section{SUDEP: Respiratory, cardiac or both?}

Our current understanding of SUDEP comes from a variety of sources. There have been measurements of the cardiorespiratory response to seizures of patients in epilepsy monitoring units (EMUs), but it remains unclear whether the observed effects are relevant to the fatal events of SUDEP. There are also data from seizure-induced death in animal models, but it is also not known if these mechanisms are the same as those in human SUDEP. The most relevant source of data comes from human SUDEP cases, including information from death scene investigations, deaths witnessed by nonmedical personnel, and deaths of patients while monitored in the hospital.

\section{The MORTEMUS Study}

It is fortunately rare for patients to die while being monitored for seizures in EMUs. However, the common practice of withdrawing anticonvulsant medications to increase the likelihood of capturing a seizure may increase the risk of SUDEP. The recent mortality in epilepsy monitoring units study (MORTEMUS) determined that out of 93, 791 monitored patients and 1771 patient years of recording there were 16 cases of definite or probable 
SUDEP (combined here as "SUDEP") and 9 cases of "near-SUDEP"16." The data from each of these cases were carefully scrutinized to document the events leading up to death. This analysis clearly documented several important findings that had previously been suggested, but had not been convincingly demonstrated: 1) 16 out of 16 SUDEP cases, and 7 of 9 near SUDEP cases occurred after a GTCS; 2) 14 of 16 SUDEP cases occurred at night; 3 ) 14 of 16 SUDEP cases were prone; 4) CPR was successful in 7 of 7 cases when started within three minutes, but was not successful in 8 of 8 cases when started after ten minutes.

There was a consistent sequence of events that occurred after each seizure that resulted in SUDEP. Rapid breathing at 18-50 breaths/min began immediately after the seizure, followed by apnea, bradycardia and PGES. A third of the cases did not recover. The others had transient return of breathing movements and the QRS complex, but the authors suggested that there may have been "possibly ineffective respiration probably aggravated by the prone position." Some patients were noted to be lying face down and died without apparent struggle, as if they were unable to arouse in response to the stimulus they were presumably subjected to of hypercapnia and hypoxia. In some cases gasping occurred transiently. Terminal apnea always occurred first and then terminal asystole. The authors concluded by saying that: "our data suggest that the main mechanism leading to SUDEP starts with an early, centrally mediated, severe alteration of both respiratory and cardiac functions after generalized tonic clonic seizures."

Some of the main limitations to the study were that: 1) There were no direct measurements of breathing, including any information on tidal volume, blood gases or $\mathrm{O} 2$ saturation; 2) The seizure itself obscured the collection of data during the ictus, including whether there was any effective breathing, and; 3) An autopsy was not obtained in 6 out of the 11 monitored SUDEP cases. Perhaps the most important of these limitations was that breathing was evaluated by visual observation of the video, with the only measurement being respiratory rate. This approach has the limitation that it is not possible to determine the tidal volume, or to rule out paradoxical breathing, airway obstruction or other ineffective respiratory effort despite normal or increased respiratory rate. As the authors point out, "respiration is withheld during GTCS, resulting in ictal hypoxaemia in $33 \%$ of cases"13, 16 . Thus, brain oxygenation might well be already compromised before postictal apnoea in GTCS-induced SUDEP." Thus, during the seizure there may be severe hypoventilation, leading to oxygen debt, hypercapnia and respiratory acidosis. Immediately after the seizure is over there may be transient restoration of cardiac and respiratory function, but it may not be effective enough to reverse the severe alteration in blood gases that occurred during the ictus. A respiratory rate of 50 per minute is very unusual and likely signifies a severe increase in respiratory drive. If there is ineffective respiration, it could be aggravated by the prone position, due to the greater strength needed to lift the body and move air. As noted, there may also be no attempt to overcome these problems, since the patient is in a "postictal state" of decreased arousal, and lack of awareness of the dangerous circumstances.

These data are unique in their scope, with so many cases of SUDEP, and despite some limitations they are unprecedented in the ability to define common factors. For example, the correlation with GTCS, night and the prone position offer windows into possible mechanisms. If $\mathrm{O}_{2}$ desaturation is just as common after seizures that do not generalize, then 
why did all SUDEP cases occur after GTCS? The results of this study and others clearly indicate that individual SUDEP cases involve both respiratory and cardiac abnormalities ${ }^{16,57-62}$. It has been very difficult for any specific SUDEP case to draw a firm conclusion about whether respiratory or cardiac dysfunction was first to appear. The observation that "terminal apnoea always preceded terminal asystole" is irrelevant to this question, because those occur long after the initiating events. In some instances both may occur at the onset, and so a combination of the two may be the primary cause of death, referred to as "neurovegetative breakdown" 16 . In other cases it is likely that respiratory dysfunction is the primary cause of death, whereas in some cases the initiating factor is probably cardiac or autonomic dysfunction.

\section{Genetics of SUDEP}

There are no genetic mutations identified that cause SUDEP, but there are a variety that have been associated with an increase in risk (Table 1). It is possible that genetic screening of families of patients who have died of SUDEP could uncover mutations and polymorphisms in unidentified genes that make it more likely for cardiorespiratory dysfunction to occur during seizures or in response to hypoxia ${ }^{63}$. There are also genes that confer an increased risk of SUDEP for reasons that are not yet clear. Examples include those for long QT syndrome (LQTS) and Dravet syndrome (DS).

\section{Long QT Syndrome}

LQTS is caused by mutations in 13 or more genes with variable penetrance. These lead to a prolonged cardiac action potential, an increased susceptibility to syncope, ventricular tachycardia, and sudden death ${ }^{64}$. The syndrome is a major cause of malignant ventricular arrhythmias and sudden death in younger individuals with otherwise normal hearts. Almost $50 \%$ of LQTS cases are caused by a mutation in the KCNQ1 gene, which encodes the a subunit of the voltage-gated potassium channel $\mathrm{K}_{\mathrm{v}} 7.1^{64}$. This mutation disrupts the slow repolarizing $I_{\mathrm{KS}}$ current, leading to the increased cardiac action potential duration ${ }^{65}$. LQTS genes are expressed throughout the body, with 10 of 13 found in the brain and heart. The majority of mutations that cause LQTS are in ion channels that are critical for normal cardiomyocyte function, and it has now become clear that some are also important for neuronal function. A recent study showed that a seizure phenotype was present in about $29 \%$ of LQTS patients ${ }^{66}$. Seizure phenotypes were more common in LQT2 (KCNH2) than LQT1 (KCNQ1) and LQT3 (SNC5A), which together make up about 75\% of all LQTS patients ${ }^{66}$. Though cardiac syncope could present as seizures, a recent study reported that $15 \%$ of LQTS patients with reported seizures had documented EEG-identified epileptiform activity ${ }^{67}$. Two mouse strains that carry mutations in Kcnql recapitulate the human cardiac phenotype ${ }^{68}$. These mice also had epileptiform EEGs compared to wildtype littermates ${ }^{69}$. Simultaneous EEG-EKG recordings demonstrated that interictal hypersynchronous cortical discharges coincided with either prolongation of cardiac RR interval and premature ventricular contractions or asystole ${ }^{69}$, which are commonly associated with autonomic instability and have been reported in SUDEP cases ${ }^{70}$. Additionally KCNA1, which encodes the Kv1.1 potassium channel, is also expressed in the brain and heart. Its deletion in mice leads to severe seizures with a high rate of sudden death ${ }^{71}$. Kv1.1 deficiency alters 
parasympathic neurotransmission and disrupts cardiac rhythmicity ${ }^{71}$. Previous research has focused on the effects of LQTS mutations on cardiac function, but it will also be important to determine whether these genes are expressed in respiratory neurons, and whether mutations could lead to changes in control of breathing, and more apnea after seizures.

\section{Dravet Syndrome}

DS is a severe childhood onset form of epilepsy, which typically manifests as febrile seizures in the first year of life and develops into refractory polymorphic seizures ${ }^{72}$. Affected children have comorbidities, including: psychomotor regression, ataxia, sleep disturbance, cognitive impairment, and sudden death. Some of these comorbidities are caused by seizures ${ }^{73}, 74$. The genetic cause of DS in $80 \%$ of cases is a loss-of-function mutation in SCN1A, which encodes the pore-forming subunit of the Nav1.1 voltage-gated sodium channel ${ }^{75,76}$. SCN1A is expressed in both the brain and heart, and plays a crucial role in the normal function of both organs ${ }^{77}$. Intrinsic cardiac abnormalities in DS are well documented. Recently progress has been made in understanding seizure-related cardiorespiratory control. A mouse model of DS recapitulates many aspects of the clinical condition and offers a research tool for understanding mechanisms of SUDEP ${ }^{78}$. Studies have reported suppressed inter-ictal resting heart rate variability and instances of ictal bradycardia that were correlated with tonic phases of GTCS ${ }^{79}$. They also report death in mice after prolonged ictal-onset bradycardia ${ }^{79}$. Muscarinic antagonists decrease bradycardia and seizure-induced death in DS mice, suggesting that SUDEP is initiated by increased vagal parasympathetic output causing lethal bradycardia ${ }^{72}$. These mouse data parallel findings from DS patients, as these patients have suppressed resting heart rate variability compared to non-DS epilepsy patients and the general population ${ }^{80}$. Although cardiac mechanisms of death have been investigated, whether respiratory dysfunction could cause SUDEP in humans or in mice with DS mutations remains unknown. It will be paramount to better understand cardiorespiratory dysfunction in DS and how that contributes to SUDEP.

\section{Cellular mechanisms}

The majority of work on the mechanisms of SUDEP have focused on cardiac electrophysiology and on cortical seizure spread. More recently, clues from animal experiments and from brain slice work point to possible brainstem mechanisms involving serotonin and adenosine, and to abnormalities of sympathetic innervation.

\section{Serotonin}

5-HT is a monoamine neurotransmitter with a variety of functions in the human brain with one of its most vital functions to stimulate breathing 81,82 . The first time that a defect in the 5-HT system was linked to seizure-induced death in mice was in 5- $\mathrm{HT}_{2 \mathrm{C}}$ receptor knockout mice that experience seizures and die prematurely from sudden death. ${ }^{22}$ The 5 -HT system was later implicated in postictal hypoventilation, with the observation that the respiratory arrest seen in DBA/2 mice during and after audiogenic seizures (see above), can be prevented with fluoxetine treatment ${ }^{83}$. Additionally, these mice have aberrant serotonin (5hydroxytryptamine; 5-HT) receptor expression in some regions of the brainstem ${ }^{84}$. These data led to the conclusion that 5-HT may be involved in the pathophysiology of SUDEP. 
These observations in mice gave rationale to a study in patients with medically refractory partial epilepsy that found selective serotonin reuptake inhibitors (SSRIs) decreased the likelihood of ictal hypoxemia ${ }^{85}$.

These results implicating a potential role of 5-HT in SUDEP are consistent with the known critical role of 5-HT in control of breathing. Normal respiratory control is dependent on neurons in the medulla and pons that generate and shape the respiratory motor output. Respiratory neurons are concentrated in the nucleus of the solitary tract (NTS), nucleus ambiguous (NA), pre-Botzinger Complex (preBotC) and other regions of the lower brainstem. Serotonin neurons project to and stimulate neurons in each of these nuclei ${ }^{21,86}$. Serotonin neurons induce pacemaker activity in neurons of the preBotC and the NTS ${ }^{81}$, presumably enhancing respiratory motor output. $L m x l b^{f f f / p}$ mice, in which essentially all 5HT neurons are genetically deleted during in utero development, suffer from apnea and high mortality during the neonatal period ${ }^{87}$, have blunted $\mathrm{CO}_{2}$ chemoreception as adults ${ }^{88}$, and a defect in arousal from sleep in response to increased $\mathrm{CO}_{2}{ }^{89}$. Furthermore DOI, a 5-HT2A/1C receptor agonist, decreases the apneic episodes and mortality seen in neonatal $L m x / b^{f f f / p}$ mice ${ }^{87}$ and exogenous 5-HT can rescue the decreased hypercapnic ventilatory response (HCVR) seen in adult $L m x 1 b^{f / f / p}$ mice 88 .

Thus, a defect in the 5-HT system could make SUDEP more likely to occur. One possible scenario is that after a seizure, abnormal function of the 5-HT system leads to an arousal defect and a blunted HCVR. Together this would cause hypoxemia and death due to failure of the patient to awaken, relieve any airway obstruction, and increase ventilation.

A defect in the 5-HT system could also explain comorbidities observed in some patients with epilepsy such as depression ${ }^{90}$. In one study, patients were given the Neurological Disorders Depression Inventory for Epilepsy (NDDI-E), and there was a correlation between higher NDDI-E scores, which denotes major depression, and frequency of seizures ${ }^{91}$. This is important because frequency of seizures is a critical predictor of SUDEP. Thus, a defect in the 5-HT system could put patients at greater risk for SUDEP. Future experiments are needed to determine if there are defects in the 5-HT system in mouse models of SUDEP, and if interventions to stimulate the 5-HT system could help reduce hypoventilation and death.

\section{Adenosine}

Adenosine is an inhibitory modulator of neuronal activity and adenosine receptors are expressed throughout the nervous system ${ }^{92}$. Activation of A1 receptors is anticonvulsive ${ }^{92}$. Adenosine deaminase (ADA) and adenosine kinase (ADK) are responsible for metabolizing adenosine $^{92}$. During seizures, adenosine levels increase in the brain and suppress seizure activity ${ }^{93}$. However, activation of adenosine receptors in the brainstem also induces severe respiratory depression ${ }^{94}$. It is possible that the increase in extracellular adenosine causes hypoventilation, apnea, and arrhythmias in the postictal period. When mice treated with pharmacological inhibitors of ADA and ADK are given kainic acid to induce seizures, they are initially protected from seizures in the first 15 minutes ${ }^{95}$. Following this initial period, the mice all develop severe seizures and die. However, if treated with caffeine (an adenosine receptor antagonist) at seizure onset, the survival time of these mice is significantly increased compared with their untreated counterparts ${ }^{95}$. By prolonging survival time with 
caffeine, Shen and colleagues ${ }^{95}$ concluded adenosine is responsible for seizure-induced death. However, caution should be taken extending these results to SUDEP for two reasons. First, these mice were given a large dose of kainic acid that induced death due to status epilepticus, which excludes a SUDEP diagnosis. Second, mice died even after caffeine treatment, which means caffeine is not an effective treatment for preventing death in this model. In the future, more research needs to be performed on the effects of caffeine on cardiorespiratory function in the peri-ictal period in mouse models with induced and spontaneous seizures that lead to sudden death in the absence of status epilepticus. However, increased adenosine remains a possible mechanism for postictal cardiorespiratory depression in epilepsy patients.

\section{Sympathetic denervation}

Data supporting alterations in sympathetic and parasympathetic tone in patients with epilepsy have been inconsistently reported, but it is postulated that there is a decrease in parasympathetic tone and an increase in sympathetic ${ }^{34,96-99}$. Studies have used ${ }^{123} \mathrm{I}$ metaiodobenzylguanidine (MIBG) to quantify cardiac sympathetic regulation in patients with epilepsy ${ }^{100-102}$. MIBG uptake into myocardial sympathetic nerve terminals was reduced in patients with chronic TLE, indicative of decreased postganglionic sympathetic innervation ${ }^{100}$. Patients with TLE that demonstrated ictal asystole also showed decreased MIBG uptake as compared to patients with TLE that did not have asystole ${ }^{101}$. In patients who underwent surgical resection, cardiac sympathetic denervation, as assessed by MIBG uptake, continued; however, denervation was greater in patients that continued to experience seizures ${ }^{102}$. Data from these MIBG studies indicate that seizures in epileptic patients could induce sympathetic denervation and autonomic dysfunction. It has been hypothesized that decreased innervation seen in these studies could induce hypersensitivity of $\beta$-adrenergic receptors in the heart ${ }^{8}$. Denervation-induced hypersensitivity could explain the increased effect of sympathetic input on heart rate, leading to tachycardia during seizures ${ }^{103}$. Increased levels of sympathetic activity can lead to secondary cardiac problems such as transient dilatation of ventricular walls, causing Takotsubo cardiomyopathy ${ }^{104}$. This pathology has been reported in GTCS and could cause cardiac dysfunction in patients with epilepsy ${ }^{105}$.

\section{Parallels to SIDS}

SUDEP and sudden infant death syndrome (SIDS) share many similarities ${ }^{6,90}$, and both are linked to defects in the 5-HT system. SIDS is defined as the death of an infant in the first year of life, which is often associated with sleep and the cause of death is not identified upon autopsy, death scene investigation, or complete review of the clinical history ${ }^{106}$. This definition is very similar to that of SUDEP. Both are diagnoses of exclusion and there are no pathognomonic features. SIDS is thought to be caused by arousal deficits and cardiorespiratory dysfunction and much of the current research is focused on investigating the role of 5-HT in arousal and breathing ${ }^{107,108}$. Available data points to a defect in the 5HT system in SIDS cases, including: increased number of 5-HT neurons of immature appearance ${ }^{109}$; reduced $5-\mathrm{HT}_{1 \mathrm{~A}}$ ligand binding in the raphe nuclei ${ }^{109}$; and reduced 5-HT in the brainstem ${ }^{110,111}$. The many similarities between the two syndromes makes it more convincing that 5-HT dysfunction may also play a role in SUDEP. Moreover, one study 
reported that infants who had a prolonged QT interval in the first week of life had a greater than 40 -fold increase in risk of SIDS than controls ${ }^{112}$, which further links SIDS and SUDEP. Since most cases of SUDEP and SIDS are unwitnessed, it is possible that some SIDS cases are actually SUDEP 6,113 .

\section{Summary and hypothesis}

The mechanisms of SUDEP remain undefined. Cardiac and respiratory abnormalities both occur quite commonly during and after seizure activity, yet SUDEP incidence is only 2000-3000 cases per year ${ }^{2}$. This disconnect between the large number of cardiorespiratory abnormalities after seizures and the much lower incidence of SUDEP could be attributable to severity of cardiorespiratory depression. In cases where the abnormality is mild, a patient would easily recover and mortality rates would be low. Conversely, in cases where a patient has prolonged cardiac or respiratory depression they would be unable to fully recover and mortality would be high. If either cardiac or respiratory dysfunction occurred in parallel with arousal deficits, the risk of SUDEP would be even higher.

We propose a hypothesis in which seizures activate neurons that project to the midbrain and medulla and inhibit monoaminergic and cholinergic neurons of the AAS, including the component that descends to the respiratory network in the medulla (Fig. 4). Activity of cardiovascular and respiratory neurons would be inhibited, at the same time that cortical activity is suppressed. At the end of the seizure, inhibition of the AAS would cause postictal unresponsiveness and PGES. Hypoventilation during the seizure would be followed by severe postictal hypercapnia and hypoxia. These blood gas derangements would then lead to bradycardia, asystole, and death (Fig. 4). Physiological monitoring of a theoretical patient during an episode of SUDEP is shown in Fig. 5, illustrating the sequence of events that might occur during a primary respiratory event, and consistent with data obtained in the MORTEMUS study. Mouse models of SUDEP may be valuable to investigate whether the same sequence of events occurs, and to then investigate cellular mechanisms such as a role of 5-HT or adenosine. Adopting new treatments will rely on identification of biomarkers for those at high risk of SUDEP and a greater understanding of underlying mechanisms.

\section{References}

1. Ficker DM, et al. Population-based study of the incidence of sudden unexplained death in epilepsy. Neurology. 1998; 51:1270-4. [PubMed: 9818844]

2. Thurman, DJ. Partners Against Mortality in Epilepsy (PAME). American Epilepsy Society; Evanston, IL: 2012.

3. Shorvon S, Tomson T. Sudden unexpected death in epilepsy. Lancet. 2011; 378:2028-38. [PubMed: 21737136]

4. Hesdorffer DC, et al. Combined analysis of risk factors for SUDEP. Epilepsia. 2011; 52:1150-9. [PubMed: 21671925]

5. Donner, EJ.; Jeffs, T.; Jette, N. Partners Against Mortality in Epilepsy (PAME). American Epilepsy Society; Evanston, IL: 2012.

6. Sowers LP, Massey CA, Gehlbach BK, Granner MA, Richerson GB. Sudden unexpected death in epilepsy: fatal post-ictal respiratory and arousal mechanisms. Respir Physiol Neurobiol. 2013; 189:315-23. [PubMed: 23707877]

7. Nashef L. Sudden unexpected death in epilepsy: terminology and definitions. Epilepsia. 1997; 38:S6-8. [PubMed: 19909329] 
8. Surges R, Thijs RD, Tan HL, Sander JW. Sudden unexpected death in epilepsy: risk factors and potential pathomechanisms. Nat Rev Neurol. 2009; 5:492-504. [PubMed: 19668244]

9. Jackson JH. On asphyxia in slight epileptic paroxysms. Lancet. 1899; 153:79-80.

10. Watanabe K, et al. Seizures with apnea in children. Pediatrics. 1982; 70:87-90. [PubMed: 7088639]

11. James MR, Marshall H, Carew-McColl M. Pulse oximetry during apparent tonic-clonic seizures. Lancet. 1991; 337:394-5. [PubMed: 1671426]

12. Nashef L, et al. Apnoea and bradycardia during epileptic seizures: relation to sudden death in epilepsy. J Neurol Neurosurg Psychiatry. 1996; 60:297-300. [PubMed: 8609507]

13. Bateman LM, Li CS, Seyal M. Ictal hypoxemia in localization-related epilepsy: analysis of incidence, severity and risk factors. Brain. 2008; 131:3239-45. [PubMed: 18952672]

14. Terrence CF, Rao GR, Perper JA. Neurogenic pulmonary edema in unexpected, unexplained death of epileptic patients. Ann Neurol. 1981; 9:458-64. [PubMed: 7271241]

15. Leestma JE, Walczak T, Hughes JR, Kalelkar MB, Teas SS. A prospective study on sudden unexpected death in epilepsy. Ann Neurol. 1989; 26:195-203. [PubMed: 2774506]

16. Ryvlin $\mathrm{P}$, et al. Incidence and mechanisms of cardiorespiratory arrests in epilepsy monitoring units (MORTEMUS): a retrospective study. Lancet Neurol. 2013; 12:966-77. [PubMed: 24012372]

17. Nelson DA, Ray CD. Respiratory arrest from seizure discharges in the limbic system. Trans Am Neurol Assoc. 1968; 93:251-2. [PubMed: 5711033]

18. Seyal M, Pascual F, Lee CY, Li CS, Bateman LM. Seizure-related cardiac repolarization abnormalities are associated with ictal hypoxemia. Epilepsia. 2011; 52:2105-11. [PubMed: 21906052]

19. Venit EL, Shepard BD, Seyfried TN. Oxygenation prevents sudden death in seizure-prone mice. Epilepsia. 2004; 45:993-6. [PubMed: 15270769]

20. Richerson GB. Response to $\mathrm{CO} 2$ of neurons in the rostral ventral medulla in vitro. J Neurophysiol. 1995; 73:933-44. [PubMed: 7608778]

21. Richerson GB. Serotonergic neurons as carbon dioxide sensors that maintain $\mathrm{pH}$ homeostasis. Nat Rev Neurosci. 2004; 5:449-61. [PubMed: 15152195]

22. Tecott LH, et al. Eating disorder and epilepsy in mice lacking 5-HT2c serotonin receptors. Nature. 1995; 374:542-6. [PubMed: 7700379]

23. Faingold CL, Randall M, Tupal S. DBA/1 mice exhibit chronic susceptibility to audiogenic seizures followed by sudden death associated with respiratory arrest. Epilepsy Behav. 2010; 17:436-40. [PubMed: 20335075]

24. Russell AE. Cessation of the pulse during the onset of epileptic fits: with remarks on the mechanism of fits. Lancet. 1906; 168:152-154.

25. Stollberger C, Finsterer J. Cardiorespiratory findings in sudden unexplained/unexpected death in epilepsy (SUDEP). Epilepsy Res. 2004; 59:51-60. [PubMed: 15135167]

26. Rowe, PC. The Harriet Lane Handbook. Medical Handbook Publishers; Chicago: 1987.

27. Moseley BD, Nickels K, Britton J, Wirrell E. How common is ictal hypoxemia and bradycardia in children with partial complex and generalized convulsive seizures? Epilepsia. 2010; 51:1219-24. [PubMed: 20067502]

28. Kiely DG, Cargill RI, Lipworth BJ. Effects of hypercapnia on hemodynamic, inotropic, lusitropic, and electrophysiologic indices in humans. Chest. 1996; 109:1215-21. [PubMed: 8625670]

29. Roche F, et al. Effect of acute hypoxia on QT rate dependence and corrected QT interval in healthy subjects. Am J Cardiol. 2003; 91:916-9. [PubMed: 12667592]

30. Moseley BD, et al. Electrocardiographic and oximetric changes during partial complex and generalized seizures. Epilepsy Res. 2011; 95:237-45. [PubMed: 21561737]

31. Stein PK, Kleiger RE. Insights from the study of heart rate variability. Annu Rev Med. 1999; 50:249-61. [PubMed: 10073276]

32. Tomson T, Ericson M, Ihrman C, Lindblad LE. Heart rate variability in patients with epilepsy. Epilepsy Res. 1998; 30:77-83. [PubMed: 9551847]

33. Ronkainen E, et al. Suppressed circadian heart rate dynamics in temporal lobe epilepsy. J Neurol Neurosurg Psychiatry. 2005; 76:1382-6. [PubMed: 16170081] 
34. Hilz MJ, Devinsky O, Doyle W, Mauerer A, Dutsch M. Decrease of sympathetic cardiovascular modulation after temporal lobe epilepsy surgery. Brain. 2002; 125:985-95. [PubMed: 11960889]

35. Dutsch M, Devinsky O, Doyle W, Marthol H, Hilz MJ. Cerebral autoregulation improves in epilepsy patients after temporal lobe surgery. J Neurol. 2004; 251:1190-7. [PubMed: 15503096]

36. Persson H, Kumlien E, Ericson M, Tomson T. Preoperative heart rate variability in relation to surgery outcome in refractory epilepsy. Neurology. 2005; 65:1021-5. [PubMed: 16217053]

37. Hartikainen JE, Malik M, Staunton A, Poloniecki J, Camm AJ. Distinction between arrhythmic and nonarrhythmic death after acute myocardial infarction based on heart rate variability, signalaveraged electrocardiogram, ventricular arrhythmias and left ventricular ejection fraction. J Am Coll Cardiol. 1996; 28:296-304. [PubMed: 8800101]

38. DeGiorgio CM, DeGiorgio AC. SUDEP heart rate variability. Epilepsy Res. 2010; 90:309-10. author reply 311-2. [PubMed: 20399076]

39. Surges R, et al. Do alterations in inter-ictal heart rate variability predict sudden unexpected death in epilepsy? Epilepsy Res. 2009; 87:277-80. [PubMed: 19747799]

40. Theodore WH, et al. The secondarily generalized tonic-clonic seizure: a videotape analysis. Neurology. 1994; 44:1403-7. [PubMed: 8058138]

41. Jobst BC, et al. Secondarily generalized seizures in mesial temporal epilepsy: clinical characteristics, lateralizing signs, and association with sleep-wake cycle. Epilepsia. 2001; 42:1279-87. [PubMed: 11737163]

42. Escueta AV, Kunze U, Waddell G, Boxley J, Nadel A. Lapse of consciousness and automatisms in temporal lobe epilepsy: a videotape analysis. Neurology. 1977; 27:144-55. [PubMed: 556830]

43. Hoffmann JM, Elger CE, Kleefuss-Lie AA. Lateralizing value of behavioral arrest in patients with temporal lobe epilepsy. Epilepsy Behav. 2008; 13:634-6. [PubMed: 18655846]

44. McPherson A, et al. Testing for minimal consciousness in complex partial and generalized tonicclonic seizures. Epilepsia. 2012; 53:e180-3. [PubMed: 22931210]

45. Penfield W. Centrencephalic integrating system. Brain. 1958; 81:231-4. [PubMed: 13572693]

46. Jasper HH. Current evaluation of the concepts of centrencephalic and cortico-reticular seizures. Electroencephalogr Clin Neurophysiol. 1991; 78:2-11. [PubMed: 1701711]

47. Moruzzi G, Magoun HW. Brain stem reticular formation and activation of the EEG. Electroencephalogr Clin Neurophysiol. 1949; 1:455-73. [PubMed: 18421835]

48. Starzl TE, Taylor CW, Magoun HW. Ascending conduction in reticular activating system, with special reference to the diencephalon. J Neurophysiol. 1951; 14:461-77. [PubMed: 14889301]

49. Azmitia EC, Gannon PJ. The primate serotonergic system: a review of human and animal studies and a report on Macaca fascicularis. Adv Neurol. 1986; 43:407-68. [PubMed: 2418648]

50. Aston-Jones G, Cohen JD. An integrative theory of locus coeruleus-norepinephrine function: adaptive gain and optimal performance. Annu Rev Neurosci. 2005; 28:403-50. [PubMed: 16022602]

51. Bromberg-Martin ES, Matsumoto M, Hikosaka O. Dopamine in motivational control: rewarding, aversive, and alerting. Neuron. 2010; 68:815-34. [PubMed: 21144997]

52. Jones BE. Activity, modulation and role of basal forebrain cholinergic neurons innervating the cerebral cortex. Prog Brain Res. 2004; 145:157-69. [PubMed: 14650914]

53. Fuller PM, Sherman D, Pedersen NP, Saper CB, Lu J. Reassessment of the structural basis of the ascending arousal system. J Comp Neurol. 2011; 519:933-56. [PubMed: 21280045]

54. Blumenfeld H. Impaired consciousness in epilepsy. Lancet Neurol. 2012; 11:814-26. [PubMed: 22898735]

55. Blumenfeld $\mathrm{H}$, et al. Positive and negative network correlations in temporal lobe epilepsy. Cereb Cortex. 2004; 14:892-902. [PubMed: 15084494]

56. Motelow, JE., et al. Society for Neuroscience. New Orleans, LA: 2012.

57. Dasheiff RM, Dickinson LJ. Sudden unexpected death of epileptic patient due to cardiac arrhythmia after seizure. Arch Neurol. 1986; 43:194-6. [PubMed: 3947265]

58. Purves SJ, Wilson-Young M, Sweeney VP. Sudden death in epilepsy: single case report with video-EEG documentation. Epilepsia. 1992; 33 (suppl 3):123. 
59. Bird JM, Dembny AT, Sandeman D, Butler S. Sudden unexplained death in epilepsy: an intracranially monitored case. Epilepsia. 1997; 38 (suppl 11):S52-S56. [PubMed: 9092961]

60. Lee HW, Hong SB, Tae WS, Seo DW, Kim SE. Partial seizures manifesting as apnea only in an adult. Epilepsia. 1999; 40:1828-31. [PubMed: 10612352]

61. McLean BN, Wimalaratna S. Sudden death in epilepsy recorded in ambulatory EEG. J Neurol Neurosurg Psychiatry. 2007; 78:1395-7. [PubMed: 17389715]

62. Bateman LM, Spitz M, Seyal M. Ictal hypoventilation contributes to cardiac arrhythmia and SUDEP: report on two deaths in video-EEG-monitored patients. Epilepsia. 2010; 51:916-20. [PubMed: 20132291]

63. Klassen TL, et al. High-resolution molecular genomic autopsy reveals complex sudden unexpected death in epilepsy risk profile. Epilepsia. 2013

64. Goldenberg I, Moss AJ. Long QT syndrome. J Am Coll Cardiol. 2008; 51:2291-300. [PubMed: 18549912]

65. Wang DW, Yazawa K, George AL Jr, Bennett PB. Characterization of human cardiac Na+ channel mutations in the congenital long QT syndrome. Proc Natl Acad Sci U S A. 1996; 93:13200-5. [PubMed: 8917568]

66. Johnson JN, et al. Identification of a possible pathogenic link between congenital long QT syndrome and epilepsy. Neurology. 2009; 72:224-31. [PubMed: 19038855]

67. Anderson JH, Bos JM, Cascino GD, Ackerman MJ. Prevalence and spectrum of electroencephalogram-identified epileptiform activity among patients with long QT syndrome. Heart Rhythm. 2014; 11:53-7. [PubMed: 24103226]

68. Casimiro MC, et al. Targeted point mutagenesis of mouse Kcnq1: phenotypic analysis of mice with point mutations that cause Romano-Ward syndrome in humans. Genomics. 2004; 84:555-64. [PubMed: 15498462]

69. Goldman AM, et al. Arrhythmia in heart and brain: KCNQ1 mutations link epilepsy and sudden unexplained death. Sci Transl Med. 2009; 1:2ra6.

70. Nei M, et al. EEG and ECG in sudden unexplained death in epilepsy. Epilepsia. 2004; 45:338-45. [PubMed: 15030496]

71. Glasscock E, Yoo JW, Chen TT, Klassen TL, Noebels JL. Kv1.1 potassium channel deficiency reveals brain-driven cardiac dysfunction as a candidate mechanism for sudden unexplained death in epilepsy. J Neurosci. 2010; 30:5167-75. [PubMed: 20392939]

72. Kalume F. Sudden unexpected death in Dravet syndrome: respiratory and other physiological dysfunctions. Respir Physiol Neurobiol. 2013; 189:324-8. [PubMed: 23850567]

73. Dravet C, Bureau M, Oguni H, Fukuyama Y, Cokar O. Severe myoclonic epilepsy in infancy: Dravet syndrome. Adv Neurol. 2005; 95:71-102. [PubMed: 15508915]

74. Oguni H, Hayashi K, Awaya Y, Fukuyama Y, Osawa M. Severe myoclonic epilepsy in infants--a review based on the Tokyo Women's Medical University series of 84 cases. Brain Dev. 2001; 23:736-48. [PubMed: 11701288]

75. Claes L, et al. De novo mutations in the sodium-channel gene SCN1A cause severe myoclonic epilepsy of infancy. Am J Hum Genet. 2001; 68:1327-32. [PubMed: 11359211]

76. Marini C, et al. Idiopathic epilepsies with seizures precipitated by fever and SCN1A abnormalities. Epilepsia. 2007; 48:1678-85. [PubMed: 17561957]

77. Maier SK, et al. An unexpected requirement for brain-type sodium channels for control of heart rate in the mouse sinoatrial node. Proc Natl Acad Sci U S A. 2003; 100:3507-12. [PubMed: 12631690]

78. Catterall WA, Kalume F, Oakley JC. NaV1.1 channels and epilepsy. J Physiol. 2010; 588:1849_ 59. [PubMed: 20194124]

79. Kalume F, et al. Sudden unexpected death in a mouse model of Dravet syndrome. J Clin Invest. 2013; 123:1798-808. [PubMed: 23524966]

80. Delogu AB, et al. Electrical and autonomic cardiac function in patients with Dravet syndrome. Epilepsia. 2011; 52 Suppl 2:55-8. [PubMed: 21463281] 
81. Ptak K, et al. Raphe neurons stimulate respiratory circuit activity by multiple mechanisms via endogenously released serotonin and substance P. J Neurosci. 2009; 29:3720-37. [PubMed: 19321769]

82. Depuy SD, Kanbar R, Coates MB, Stornetta RL, Guyenet PG. Control of breathing by raphe obscurus serotonergic neurons in mice. J Neurosci. 2011; 31:1981-90. [PubMed: 21307236]

83. Tupal S, Faingold CL. Evidence supporting a role of serotonin in modulation of sudden death induced by seizures in DBA/2 mice. Epilepsia. 2006; 47:21-6. [PubMed: 16417527]

84. Uteshev VV, Tupal S, Mhaskar Y, Faingold CL. Abnormal serotonin receptor expression in DBA/2 mice associated with susceptibility to sudden death due to respiratory arrest. Epilepsy Res. 2010; 88:183-8. [PubMed: 20018491]

85. Bateman LM, Li CS, Lin TC, Seyal M. Serotonin reuptake inhibitors are associated with reduced severity of ictal hypoxemia in medically refractory partial epilepsy. Epilepsia. 2010; 51:2211-4. [PubMed: 20491872]

86. Hodges MR, Richerson GB. The role of medullary serotonin (5-HT) neurons in respiratory control: contributions to eupneic ventilation, $\mathrm{CO} 2$ chemoreception, and thermoregulation. J Appl Physiol. 2010; 108:1425-32. [PubMed: 20133432]

87. Hodges MR, Wehner M, Aungst J, Smith JC, Richerson GB. Transgenic mice lacking serotonin neurons have severe apnea and high mortality during development. J Neurosci. 2009; 29:10341-9. [PubMed: 19692608]

88. Hodges MR, et al. Defects in breathing and thermoregulation in mice with near-complete absence of central serotonin neurons. J Neurosci. 2008; 28:2495-2505. [PubMed: 18322094]

89. Buchanan GF, Richerson GB. Central serotonin neurons are required for arousal to $\mathrm{CO}_{2}$. Proc Natl Acad Sci USA. 2010; 107:16354-16359. [PubMed: 20805497]

90. Richerson GB, Buchanan GF. The serotonin axis: Shared mechanisms in seizures, depression, and SUDEP. Epilepsia. 2011; 52:28-38. [PubMed: 21214537]

91. Dias R, et al. Depression in epilepsy is associated with lack of seizure control. Epilepsy Behav. 2010; 19:445-7. [PubMed: 20888306]

92. Boison D. Adenosine kinase, epilepsy and stroke: mechanisms and therapies. Trends Pharmacol Sci. 2006; 27:652-8. [PubMed: 17056128]

93. During MJ, Spencer DD. Adenosine: a potential mediator of seizure arrest and postictal refractoriness. Ann Neurol. 1992; 32:618-24. [PubMed: 1449242]

94. Barraco RA, Janusz CA, Schoener EP, Simpson LL. Cardiorespiratory function is altered by picomole injections of $5^{\prime}$-N-ethylcarboxamidoadenosine into the nucleus tractus solitarius of rats. Brain Res. 1990; 507:234-46. [PubMed: 2337763]

95. Shen HY, Li T, Boison D. A novel mouse model for sudden unexpected death in epilepsy (SUDEP): role of impaired adenosine clearance. Epilepsia. 2010; 51:465-8. [PubMed: 19674057]

96. Diehl B, Diehl RR, Stodieck SR, Ringelstein EB. Spontaneous oscillations in cerebral blood flow velocities in middle cerebral arteries in control subjects and patients with epilepsy. Stroke. 1997; 28:2457-9. [PubMed: 9412632]

97. Evrengul H, et al. Time and frequency domain analyses of heart rate variability in patients with epilepsy. Epilepsy Res. 2005; 63:131-9. [PubMed: 15777689]

98. Dutsch M, Hilz MJ, Devinsky O. Impaired baroreflex function in temporal lobe epilepsy. J Neurol. 2006; 253:1300-8. [PubMed: 17041741]

99. Mukherjee S, et al. Cardiovascular autonomic functions in well-controlled and intractable partial epilepsies. Epilepsy Res. 2009; 85:261-9. [PubMed: 19409754]

100. Druschky A, et al. Interictal cardiac autonomic dysfunction in temporal lobe epilepsy demonstrated by [(123)I]metaiodobenzylguanidine-SPECT. Brain. 2001; 124:2372-82. [PubMed: 11701592]

101. Kerling F, et al. Relation between ictal asystole and cardiac sympathetic dysfunction shown by MIBG-SPECT. Acta Neurol Scand. 2009; 120:123-9. [PubMed: 19154536]

102. Hilz MJ, et al. Outcome of epilepsy surgery correlates with sympathetic modulation and neuroimaging of the heart. J Neurol Sci. 2003; 216:153-62. [PubMed: 14607317] 
103. Blumhardt LD, Smith PE, Owen L. Electrocardiographic accompaniments of temporal lobe epileptic seizures. Lancet. 1986; 1:1051-6. [PubMed: 2871334]

104. Akashi YJ, Goldstein DS, Barbaro G, Ueyama T. Takotsubo cardiomyopathy: a new form of acute, reversible heart failure. Circulation. 2008; 118:2754-62. [PubMed: 19106400]

105. Chin PS, Branch KR, Becker KJ. Postictal neurogenic stunned myocardium. Neurology. 2005; 64:1977-8. [PubMed: 15955960]

106. Krous HF, et al. Sudden infant death syndrome and unclassified sudden infant deaths: a definitional and diagnostic approach. Pediatrics. 2004; 114:234-8. [PubMed: 15231934]

107. Hunt CE, Brouillette RT. Sudden infant death syndrome: 1987 perspective. J Pediatr. 1987; 110:669-78. [PubMed: 3553512]

108. Kinney HC, Richerson GB, Dymecki SM, Darnall RA, Nattie EE. The brainstem and serotonin in the sudden infant death syndrome. Annu Rev Pathol. 2009; 4:517-50. [PubMed: 19400695]

109. Paterson DS, et al. Multiple serotonergic brainstem abnormalities in sudden infant death syndrome. JAMA. 2006; 296:2124-32. [PubMed: 17077377]

110. Kinney HC, et al. Serotonergic brainstem abnormalities in Northern Plains Indians with the sudden infant death syndrome. J Neuropathol Exp Neurol. 2003; 62:1178-91. [PubMed: 14656075]

111. Duncan JR, et al. Brainstem serotonergic deficiency in sudden infant death syndrome. JAMA. 2010; 303:430-7. [PubMed: 20124538]

112. Schwartz PJ, et al. Prolongation of the QT interval and the sudden infant death syndrome. N Engl J Med. 1998; 338:1709-14. [PubMed: 9624190]

113. Kinney HC, et al. Sudden death, febrile seizures, and hippocampal and temporal lobe maldevelopment in toddlers: a new entity. Pediatr Dev Pathol. 2009; 12:455-63. [PubMed: 19606910] 


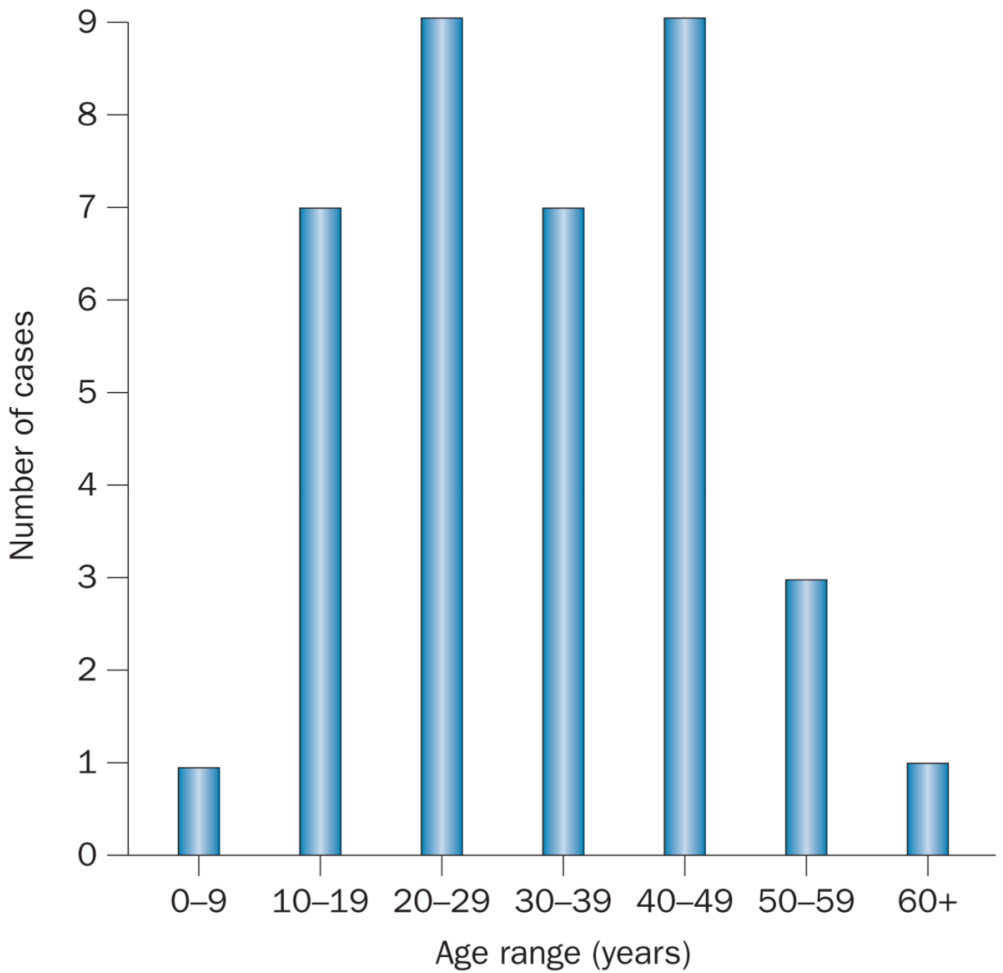

Figure 1. Distribution of SUDEP cases by age

The peak age of SUDEP incidence occurs at 30 years old. 
a
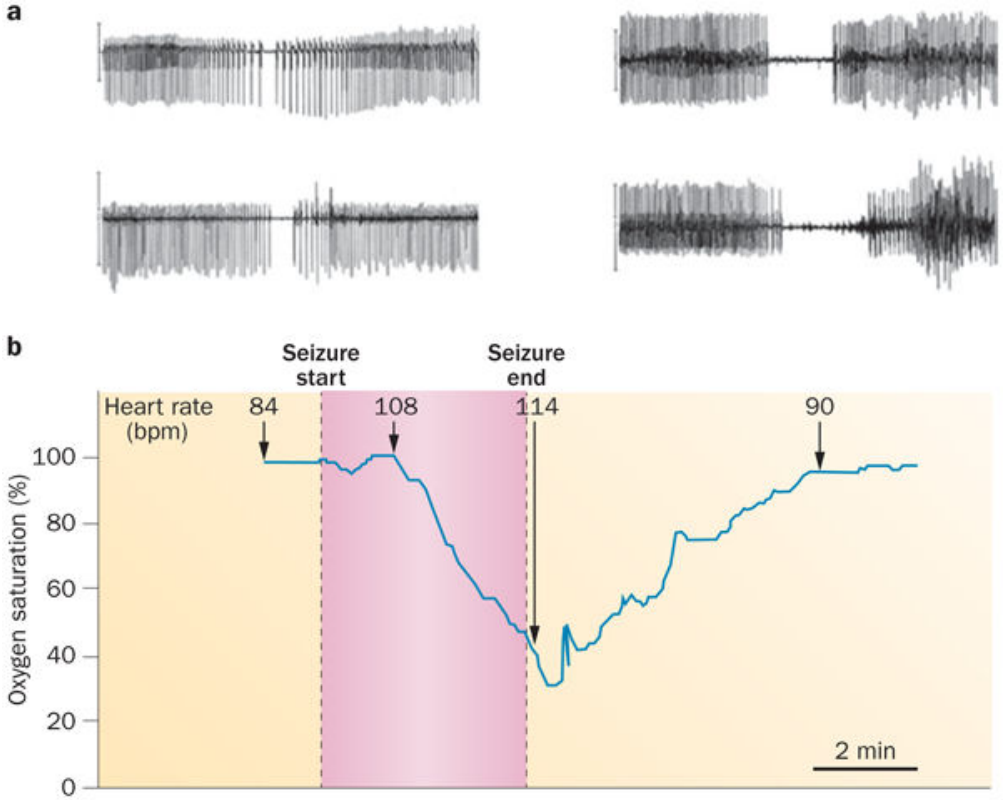

Figure 2. Cardiac and respiratory abnormalities commonly occur following seizure activity in mice and humans

A) EEG (black) and EKG (blue) traces from Dravet syndrome mice that died prematurely illustrating bradycardia following a seizure. Modified with permissions from Kalume et al., 2013. B) Oxygen saturation drops below 40\% during a partial seizure in a 19-year-old male patient. Patient was awake and sitting in bed during the hypoxic event. Seizure duration is marked by bottom arrows. Heart rate is marked by the upper arrows and beats per minute are given. Modified with permission from Bateman et al., 2008. 


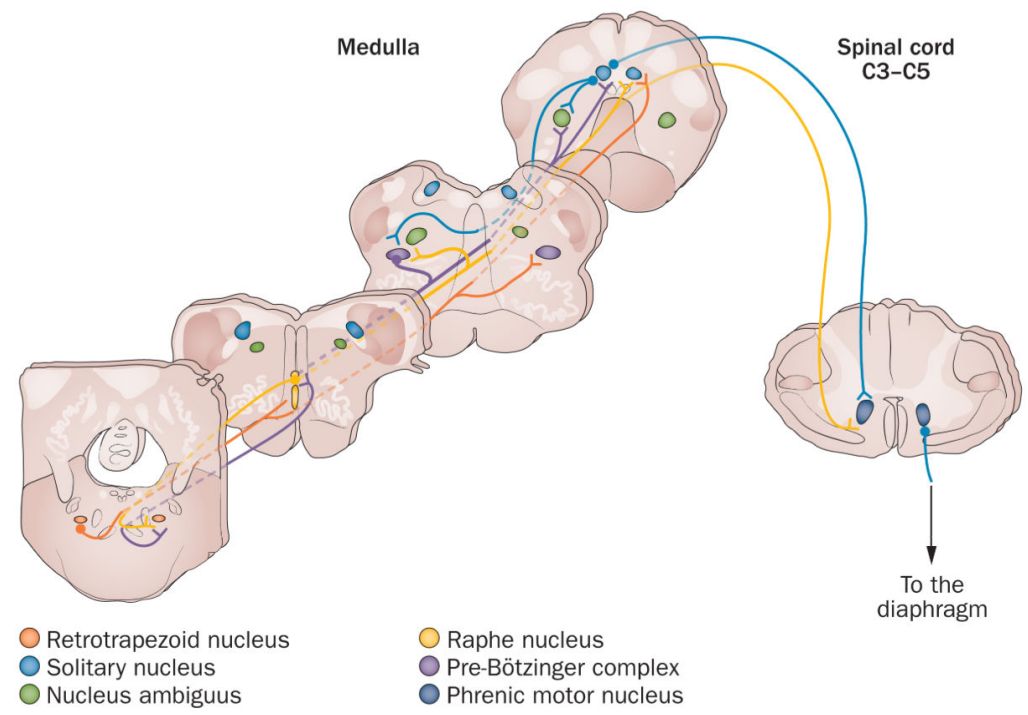

Figure 3. Anatomical distribution of arousal networks and brain stem nuclei that could be affected by seizure

A) Seizure in forebrain activates anatomical connections leading to dysfunction of brainstem nuclei critical for cardiorespiratory control. PreBotC: Pre-Botzinger complex, BotC:

Botzinger complex, NTS: Nucleus tractus solitaries, NA: Nucleus ambiguous. B) Seizure spread into the midbrain disrupts both ascending and descending arousal systems. TMN : tuberomammillary nucleus, LDT: Laterodorsal tegmental nucleus, PPT: pedunculopontine tegmental nucleus, LC: Locus coeruleus. 


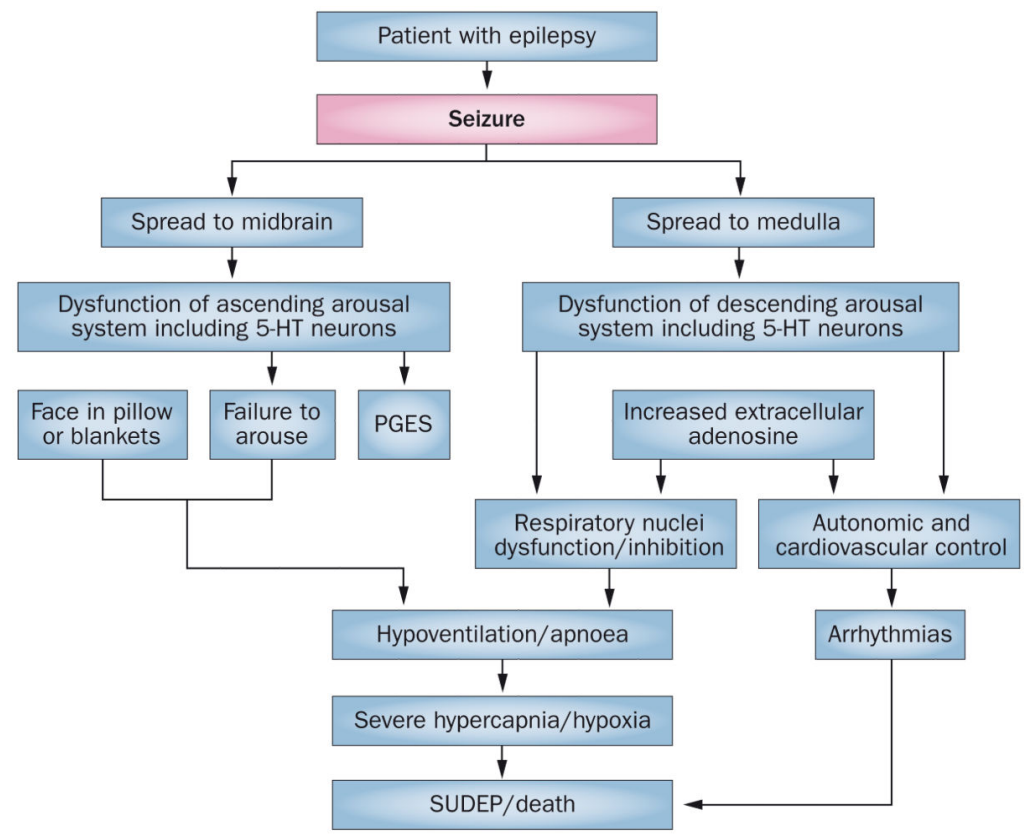

Figure 4. Hypothesized model for SUDEP

A patient with epilepsy has a seizure which spreads to both the midbrain and medulla. Spread to the midbrain causes acute dysfunction of the ascending arousal system, including 5-HT neurons, which leads to PGES and arousal failure. When combined with the face in pillows or bedding, the arousal failure leads to hypoventilation. Seizure spread into the medulla causes disruption of descending arousal system. This, along with increased extracellular adenosine, precipitates dyfunction in both respiratory nuclei and autonomic and cardiovascular control. Hypoventilation, apnea, and arrhythmias develop and lead to severe hypercapnia, hypoxia, and death. 

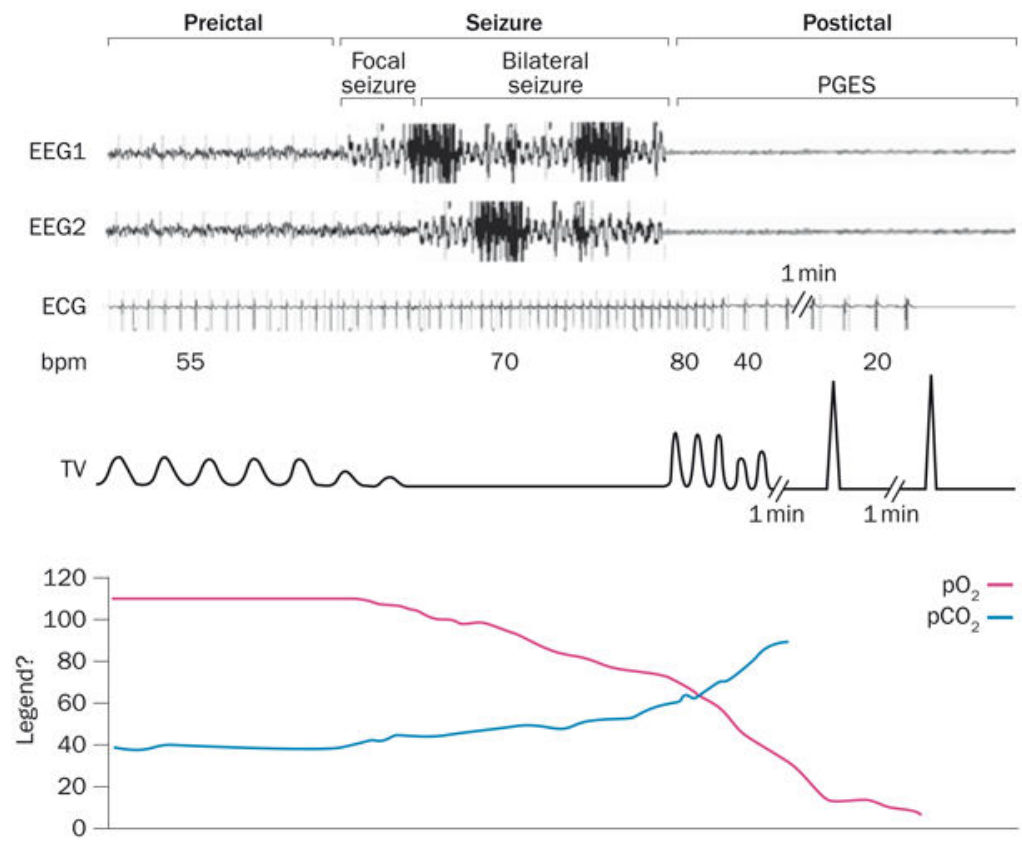

Figure 5. Proposed sequence of events surrounding a respiratory death

Normal vitals and EEG during the pre-ictal state. Seizure begins as a focal seizure and tachycardia quickly follows. Upon seizure generalization, ictal apnea occurs and pO2 begins to fall. Tachycardia continues until the end of the seizure while the pO2 continues to drop and the pCO2 begins to rise during the seizure. In the post-ictal state, PGES occurs and there is bradycardia which worsens over the course of several minutes. Tachypnea occurs due to decreased pO2 and increased pCO2 but respiratory activity decreases and apnea occurs again. pO2 drops to dangerously low levels and the patient becomes severely hypoxic and hypercapnic. Terminal gasps occur, but auto-resuscitation fails. Finally, the patient's heart stops and death quickly follows. 


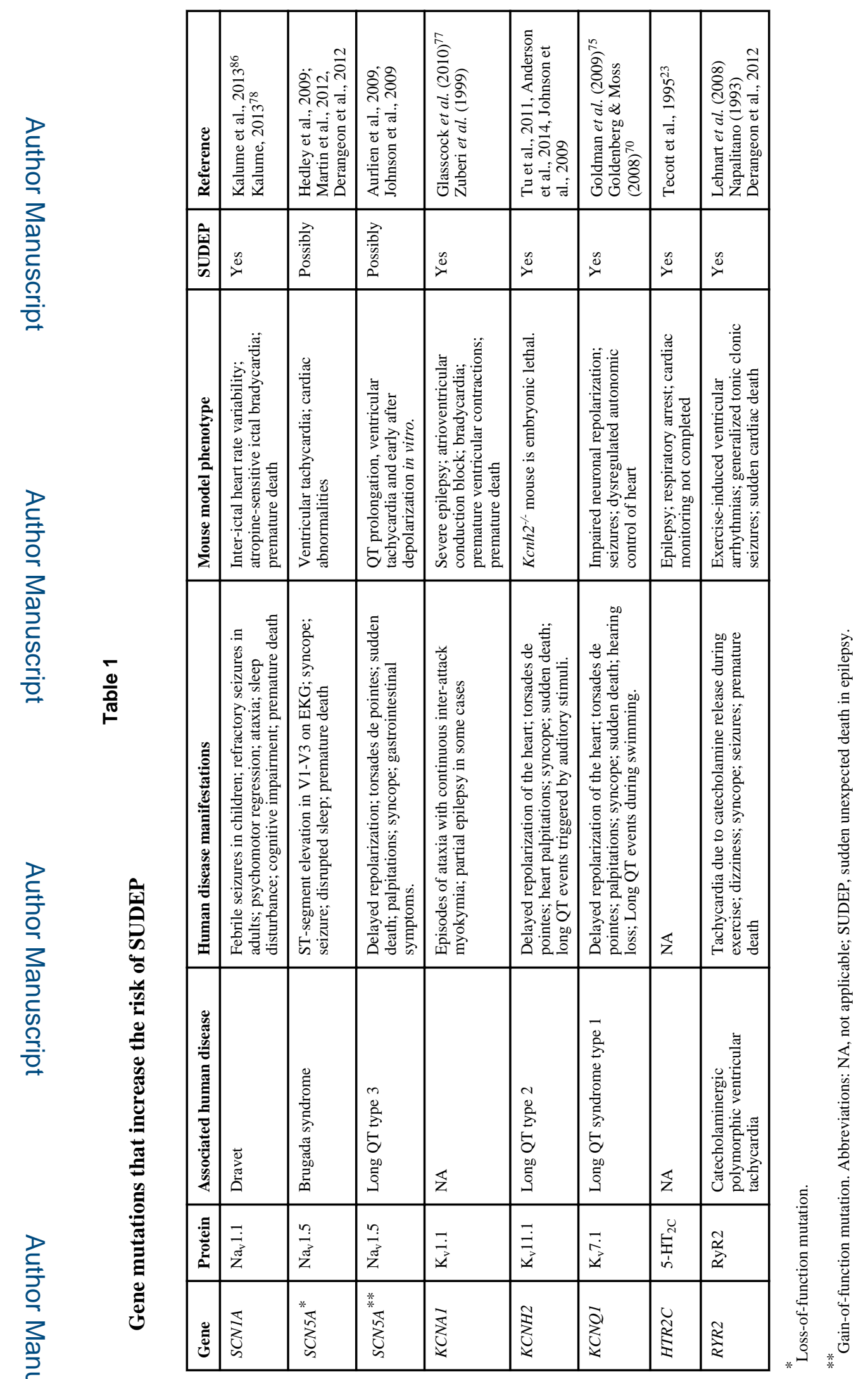

Nat Rev Neurol. Author manuscript; available in PMC 2015 September 10. 